\title{
Ửng dụng mô hình ARDL đánh giá mối quan hệ giữa thất nghiệp và tăng trưởng kinh tế ở Việt Nam
}

\section{Application of ARDL model for examining the relationship between unemployment and economic growth in Vietnam}

\author{
Nguyễn Thị Thu Hà ${ }^{*}$ \\ ${ }^{1}$ Trường Đại học Kinh tế, Đại học Đà Nẵng, Việt Nam \\ *Tác giả liên hệ, Email: ha.ntt@due.edu.vn
}

THÔNG TIN

DOI: $10.46223 / \mathrm{HCMCOUJS}$. econ.vi.16.3.959.2021

Ngày nhận: 06/09/2020

Ngày nhận lại: 31/12/2020

Duyệt đăng: 27/01/2021

Tù khóa:

định luật Okun; mối quan hệ; tăng trưởng kinh tế; thất nghiệp

Keywords:

Okun's law; the relationship; economic growth; unemployment

\section{TÓM TẮT}

Mục tiêu của nghiên cứu là phân tích mối quan hệ giữa thất nghiệp và tăng trưởng kinh tế ở Việt Nam trong giai đoạn 1986 2019. Sử dụng mô hình phân phối trễ tự hồi quy (Autoregressive Distributed Lag - ARDL) kết hợp với phương pháp kiểm định đường bao (Bound test) làm cơ sở xác định tác động dài hạn, sau đó dùng mô hình hiệu chỉnh sai số (ECM) để phân tích tác động ngắn hạn, kết quả thực nghiệm đã chứng minh được mối quan hệ dài hạn và ngắn hạn giữa thất nghiệp và tăng trưởng kinh tế. Cụ thể, thất nghiệp có tác động tiêu cực đến tăng trưởng kinh tế trong cả ngắn hạn và dài hạn, nhưng giữa hai biến vĩ mô này không tồn tại quan hệ nhân quả. Dựa trên kết quả nghiên cứu, bài viết đề xuất một số khuyến nghị nhằm hài hòa mối quan hệ này.

\section{ABSTRACT}

The paper aims to analyze the relationship between unemployment and economic growth in Vietnam during 1986 2019. The Autoregressive Distributed Lag (ARDL) was used to combine with the Bound tests to determine the long-run effects and the Error Correction Model (ECM) afterward to analyze the short-run effects. The empirical results reveal the long and shortrun linkages between unemployment and economic growth. Particularly, unemployment negatively impacts economic growth in both the short and long run, but there is no causal relationship between these two macro variables. Based on the research findings, some recommendations are proposed to harmonize this relationship.

\section{1. Đặt vấn đề}

Thất nghiệp là một trong những biến số quan trọng cần được xem xét để hiểu các động thái của nền kinh tế và từ đó xây dựng các kế hoạch chiến lược nhằm ổn định nền kinh tế cũng như thúc đẩy tăng trưởng và phát triển kinh tế. Thất nghiệp được coi là một tình huống tồi tệ nhất mà xã hội loài người có thể trải qua vì nó ảnh hưởng đến kinh tế và xã hội theo nhiều khía cạnh và hướng khác nhau (Habees \& Rumman, 2012). Tương tự, Adarkwa, Donkor, và Kyei (2017) cũng cho rằng thất nghiệp là một tình trạng nghiêm trọng mà hầu hết các quốc gia phải đối mặt, gây ra một loạt các vấn đề kinh tế và xã hội như giảm nguồn thu của chính phủ dưới dạng thuế thu nhập, lãng phí giờ làm việc và các tệ nạn như trộm cướp, mại dâm, ... 
Bản chất của thất nghiệp phụ thuộc vào cấu trúc quốc gia và loại hình quốc gia đó thuộc loại phát triển, đang phát triển hay chưa phát triển (Soylu, Çakmak, \& Okur, 2017). Anghel, Anghelache, và Manole (2017) giải thích rằng thất nghiệp như một chỉ số kinh tế vĩ mô cho thấy mức độ yếu kém của một quốc gia trong việc sử dụng nguồn lao động dồi dào của mình. Điều này cho thấy có nhiều người lao động năng động luôn sẵn sàng, tìm kiếm và có khả năng bổ sung vào thị trường lao động nhưng không thể tìm được việc làm (Yilmaz, 2005).

Tăng trưởng kinh tế được định nghĩa là sự gia tăng trong Tổng sản phẩm quốc nội (GDP) của một quốc gia, được đánh giá như là những đóng góp của người dân tương ứng với thu nhập hoặc vốn của quốc gia (Piketty, 2014). Theo Jhingan (2003), tăng trưởng kinh tế là quá trình mà ở đó thu nhập bình quân thực tế của mỗi người dân của một quốc gia tăng lên trong một khoảng thời gian dài và được xác định thông qua sự gia tăng sản lượng (hàng hóa và dịch vụ được sản xuất) trong quốc gia đó. Tăng trưởng kinh tế được coi là một trong những công cụ tài khóa tối quan trọng để giảm nghèo, giảm chênh lệch về phân phối thu nhập giữa mọi cá nhân trong xã hội cũng như cải thiện chất lượng cuộc sống người dân, gia tăng sự thịnh vượng của quốc gia (Ademola \& Badiru, 2016; Dfid, 2008).

Mối quan hệ giữa thất nghiệp và tăng trưởng kinh tế (Tổng sản phẩm quốc nội) từ lâu đã là một vấn đề quan tâm của hầu hết các nền kinh tế (Nguyen, 2016). Giảm tỷ lệ thất nghiệp và tăng tốc độ tăng trưởng kinh tế của một quốc gia là một trong những mục tiêu kinh tế vĩ mô thiết yếu mà các quốc gia phải đối mặt trong những thập kỷ gần đây (Daly \& Hobijn, 2010; Soylu et al., 2017). Trong việc hạn chế thất nghiệp, tăng trưởng kinh tế là một chỉ số kinh tế vĩ mô quan trọng có thể giúp giải quyết mối đe dọa của thất nghiệp. Phát triển kinh tế tạo cơ hội cho các doanh nghiệp mới ra đời, từ đó tạo ra việc làm, giảm tình trạng thất nghiệp và giảm nghèo ở một quốc gia. Do đó, hầu hết các quốc gia đang cố gắng giảm tỷ lệ thất nghiệp thông qua các kế hoạch chính sách tìm cách gia tăng mong muốn của các doanh nhân nhằm tạo thêm việc làm để quốc gia đó đạt được sự tiến bộ kinh tế lớn hơn (Habees \& Rumman, 2012).

Hiện tại có rất nhiều nghiên cứu cả về mặt lý thuyết và thực nghiệm đánh giá về mối quan hệ giữa thất nghiệp và tăng trưởng kinh tế ở các quốc gia khác nhau nhưng các kết quả nghiên cứu là mâu thuâ̂n, không có sự thống nhất. Điều này đang gây khó khăn cho các nhà nghiên cứu trong việc khái quát hóa các phát hiện của các nghiên cứu để từ đó đưa ra dự đoán về mối quan hệ này cho các nền kinh tế khác nhau (Seth, John, \& Dalhatu, 2018).

Theo báo cáo của Tổng cục Thống kê, Việt Nam đạt được mức tăng trưởng GDP hàng năm khoảng 7\% trong thập kỷ qua, đây được xem là một sự cải thiện lớn đối với sự phát triển kinh tế của đất nước so với các thập kỷ trước. Yếu tố góp phần đóng góp vào thành tựu này là sự gia tăng dân số của Việt Nam, điều này vô tình tạo ra nguồn nhân lực dồi dào với các kỹ năng và năng lực chuyên môn đa dạng - nhân tố làm tăng năng suất và sản lượng của mọi quốc gia, nhưng lại gây ra tác động lớn đến tình trạng thất nghiệp ở nước ta. Liệu rằng có tồn tại mối quan hệ giữa thất nghiệp và tăng trưởng kinh tế ở Việt Nam không? Hơn nữa, ở Việt Nam đến nay vẫn chưa có một nghiên cứu định lượng nào đánh giá mối quan hệ giữa thất nghiệp và tăng trưởng kinh tế dựa trên khung lý thuyết của Định luật Okun. Do đó, việc nghiên cứu ảnh hưởng của thất nghiệp đến tăng trưởng kinh tế Việt Nam là cấp thiết, điều này sẽ góp phần lấp đầy khoảng trống các nghiên cứu hiện có, đồng thời là tài liệu tham khảo cho các nhà hoạch đưa ra các chính sách vĩ mô nhằm giải quyết hài hòa mối quan hệ này.

Mục tiêu chính của nghiên cứu nhằm đánh giá mối quan hệ giữa thất nghiệp và tăng trưởng kinh tế ở Việt Nam trong cả ngắn hạn và dài hạn và xác định quan hệ nhân quả giữa hai biến vĩ mô này. Từ đó, một số khuyến nghị được đưa ra nhằm giải quyết hài hòa mối quan hệ giữa thất nghiệp và tăng trưởng kinh tế ở Việt Nam. 


\section{Cơ sở lý thuyết}

\section{1. Địh luật Okun}

Định luật Okun (1962) giải thích mối quan hệ tồn tại giữa các biến thể trong tỷ lệ thất nghiệp và các biến thể trong tăng trưởng kinh tế (GDP) để xác định sản lượng tiềm năng. Sản lượng tiềm năng là mức cao nhất của đầu ra GDP thực tế tại điểm việc làm tối đa và Định luật Okun là phép đo lường của đầu ra này liên quan đến đầu vào vốn con người. Định luật Okun mặc định rằng GDP tăng cao hơn $3 \%$ trên mức trung bình là cần thiết để giảm tỷ lệ thất nghiệp, tuy nhiên, tỷ lệ thất nghiệp này không cố định mà phụ thuộc vào tốc độ tăng trưởng sức lao động và sản lượng việc làm của quốc gia. Bên cạnh đó, Định luật Okun cũng chỉ ra rằng tỷ lệ thất nghiệp giảm $1 \%$ thì tổng sản phẩm quốc nội (GDP) tăng 3\%. Ngoài ra, Định luật Okun cũng chỉ ra hai mối quan hệ thực nghiệm đáng kể giữa tăng trưởng kinh tế và thất nghiệp đó là: sự thay đổi hàng quý trong tỷ lệ thất nghiệp có liên quan đến tăng trưởng GDP thực tế hàng quý và sự sai lệch của tỷ lệ thất nghiệp từ mức lạm phát không tăng tốc có liên quan đến sự sai lệch của GDP từ mức cao nhất (Daly \& Hobijn, 2010).

\subsection{Các nghiên cúu thục nghiệm}

Có rất nhiều các nghiên cứu thực nghiệm xem xét mối quan hệ giữa thất nghiệp và tăng trưởng kinh tế của một quốc gia. Tuy nhiên, các phát hiện là mâu thuẫn do các yếu tố như nguồn dữ liệu cho nghiên cứu, kỹ thuật kinh tế lượng được áp dụng trong nghiên cứu, ...

Vào giữa thế kỷ 20, Harrod và Domar (1940) đã thực hiện những nghiên cứu đầu tiên về mối quan hệ giữa tỷ lệ thất nghiệp và tăng trưởng kinh tế (GDP) (Nguyen, 2016). Các nghiên cứu nhấn mạnh vào các khía cạnh rối loạn chức năng có thể xảy ra của tăng trưởng kinh tế, ví dụ như những cách thức mà tăng trưởng kinh tế có thể song hành với tỷ lệ thất nghiệp ngày càng gia tăng. Solow (1956) trong nghiên cứu về sự đóng góp của lý thuyết tăng trưởng kinh tế chỉ ra rằng các nghiên cứu của Harrod và Domar (1940) dựa trên những vấn đề quan trọng của trạng thái cân bằng, bao gồm việc đánh giá giữa tỷ lệ tăng trưởng danh nghĩa - phụ thuộc vào sự gia tăng nguồn nhân lực và tốc độ tăng trưởng kinh tế đảm bảo - phụ thuộc vào hành vi tiết kiệm và đầu tư của các hộ gia đình và các công ty.

Mô hình Solow đưa các quan niệm mới vào cuộc tranh luận về tăng trưởng kinh tế bằng cách loại bỏ mối quan hệ giữa tiến bộ kinh tế và tỷ lệ thất nghiệp như là yếu tố ngoại vi (Nguyen, 2016). Solow (1956) chấp nhận tất cả các giả định khác được quy định bởi Mô hình Harrod và Domar (1940) về tăng trưởng kinh tế ngoại trừ tỷ lệ cố định sẽ dẫn đến rối loạn chức năng của tăng triển kinh tế trong dài hạn (Schiliro, 2017). Do đó, mô hình Solow giả định rằng tổng sản phẩm quốc nội (GDP) được sản xuất theo công nghệ hàm sản xuất chung.

Seth và cộng sự (2018) áp dụng kiểm định đồng liên kết độ trễ phân phối tự hồi quy (The Autoregressive Distributed Lagged (ARDL)) cho dữ liệu tỷ lệ thất nghiệp và tăng trưởng kinh tế ở Nigeria giai đoạn 1986 - 2015. Nghiên cứu không tìm thấy mối quan hệ dài hạn, nhưng tìm thấy mối quan hệ ngắn hạn thuận chiều giữa tỷ lệ thất nghiệp và tăng trưởng kinh tế ở Nigeria, cụ thể, tỷ lệ thất nghiệp tăng $1 \%$ dẫn đến tăng trưởng kinh tế (tổng sản phẩm quốc nội thực tế) tăng $20.6 \%$.

Gyang, Anzaku, và Iyakwari (2018) sử dụng các kiểm định đồng liên kết Johansen và nhân quả Granger để đánh giá mối quan hệ giữa tỷ lệ thất nghiệp, tỷ lệ lạm phát và tăng trưởng kinh tế ở Nigeria giai đoạn 1986 - 2015. Kết quả nghiên cứu chỉ ra rằng tồn tại mối quan hệ ngắn hạn và dài hạn giữa tỷ lệ thất nghiệp, tỷ lệ lạm phát và tăng trưởng kinh tế, nhưng kiểm định nhân quả Granger cho thấy thất nghiệp và lạm phát không có ý nghĩa thống kê trong việc giải 
thích tăng trưởng kinh tế ở Nigeria.

Kreishan (2011) sử dụng kiểm định đồng liên kết và hồi quy đơn giản để phân tích mối quan hệ giữa thất nghiệp và tăng trưởng kinh tế ở Jordan giai đoạn 1970 - 2008 và xem xét Định luật Okun có đúng trong trường hợp của Jordan không. Kết quả nghiên cứu cho thấy tăng trưởng kinh tế không ảnh hưởng đến thất nghiệp và không tồn tại mối quan hệ giữa tăng trưởng kinh tế và thất nghiệp ở Jordan. Định luật Okun về mối quan hệ tiêu cực giữa tăng trưởng kinh tế và thất nghiệp là không đúng trong trường hợp của Jordan.

Levine (2013) lập luận rằng mối quan hệ giữa tốc độ phát triển kinh tế và tỷ lệ thất nghiệp có thể lỏng lẻo trong ngắn hạn bởi vì không phải là hiếm khi tỷ lệ thất nghiệp thể hiện sự suy giảm bền vững, nghĩa là đôi khi sau các biện pháp, các kế hoạch chính sách kinh tế mang lại những kết quả tích cực cho thất nghiệp, nhưng điều này trái lại thường gây ra sự suy giảm, tụt hậu kinh tế. Về mặt thực nghiệm, nghiên cứu tìm thấy mối quan hệ tiêu cực giữa thất nghiệp và tăng trưởng kinh tế trong dài hạn và mối quan hệ này được giải thích rằng chừng nào sự tăng trưởng của GDP thực tế vượt qua sự tăng trưởng về năng suất lao động, thì khu vực dịch vụ sẽ gia tăng. Điều này sẽ tạo cơ hội việc làm cho lao động trong lĩnh vực sản xuất này và cuối cùng sẽ góp phần giảm tỷ lệ thất nghiệp trong dài hạn.

Özel, Sezgin, và Topkaya (2013) thực hiện một nghiên cứu đánh giá mối quan hệ giữa thất nghiệp và tăng trưởng kinh tế sử dụng dữ liệu tăng trưởng tài khóa, năng suất và thất nghiệp của 07 quốc gia công nghiệp (G7) giai đoạn 2000 - 2011. Kết quả nghiên cứu chỉ ra mối quan hệ tiêu cực giữa tăng trưởng kinh tế và thất nghiệp giai đoạn 2000 - 2007, được đánh dấu là thời kỳ tiền khủng hoảng. Thêm vào đó, nghiên cứu tìm thấy mối quan hệ tiêu cực không đáng kể và lỏng lẻo giữa tỷ lệ thất nghiệp và tốc độ tăng trưởng kinh tế giai đoạn $2008-2011$, được đánh dấu là thời kỳ hậu khủng hoảng.

Soylu và cộng sự (2017) sử dụng các kiểm định mô hình hồi quy dữ liệu bảng (Pooled Panel OLS) và đồng liên kết Johansen để đánh giá mối quan hệ giữa tăng trưởng kinh tế và tỷ lệ thất nghiệp ở các nước Đông Âu giai đoạn 1992 - 2014. Nghiên cứu tìm thấy sự hiện diện của mối quan hệ đồng liên kết tiêu cực giữa thất nghiệp và tăng trưởng kinh tế trong dài hạn. Tuy nhiên, trong ngắn hạn tăng trưởng kinh tế ảnh hưởng tích cực đến tỷ lệ thất nghiệp.

Banda, Ngirande, và Hogwe (2016) sử dụng dữ liệu chuỗi thời gian để phân tích tác động của phát triển kinh tế đến thất nghiệp ở Nam Phi giai đoạn 1994 - 2012. Nghiên cứu tìm thấy mối quan hệ tích cực giữa thất nghiệp và phát triển kinh tế trong dài hạn sau khi kiểm định đồng liên kết Johansen và ước lượng Mô hình sửa lỗi vector (Vector Error Correction Model (VECM)). Điều này có nghĩa là sự gia tăng tỷ lệ thất nghiệp trong dài hạn cũng sẽ phản ánh sự tăng trưởng kinh tế ở Nam Phi. Tương tự, nghiên cứu của Enejoh và Tsauni (2017) cũng chỉ ra mối quan hệ tích cực giữa tỷ lệ thất nghiệp và tăng trưởng kinh tế trong cả ngắn hạn và dài hạn, và hướng của quan hệ nhân quả chạy từ thất nghiệp đến tăng trưởng kinh tế.

Nghiên cứu của Li và Liu (2012) về mối quan hệ giữa tỷ lệ thất nghiệp, phát triển kinh tế và lạm phát ở Trung Quốc sử dụng dữ liệu hàng năm giai đoạn 1978 - 2010. Nghiên cứu tìm thấy mối quan hệ giữa thất nghiệp, tăng trưởng kinh tế và lạm phát trong cả ngắn hạn và dài hạn và tăng trưởng kinh tế ảnh hưởng tích cực đến tỷ lệ thất nghiệp, điều này là trái ngược với Định luật Okun. Thêm vào đó, kiểm định quan hệ nhân quả Granger chỉ thấy sự hiện diện của quan hệ một chiều chạy từ tăng trưởng kinh tế đến thất nghiệp.

Haririan, Bilgin, và Karabulut (2010) xem xét mối quan hệ giữa tổng sản phẩm quốc nội (GDP) (tăng trưởng kinh tế) và tỷ lệ thất nghiệp ở các quốc gia MENA (Thổ Nhĩ Kỳ, Ai Cập, Israel và Jordan). Nghiên cứu xác nhận sự hiện diện của mối quan hệ dài hạn giữa tăng trưởng 
GDP và tỷ lệ thất nghiệp và tiếp tục khẳng định mối quan hệ tiêu cực giữa GDP và tỷ lệ thất nghiệp, nghĩa là, tăng trưởng GDP dẫn đến giảm tỷ lệ thất nghiệp ở các quốc gia MENA.

Khaliq, Soufan, và Shihab (2014) sử dụng kiểm định Pooled EGLS (Cross-section SUR) để kiểm tra mối quan hệ giữa tỷ lệ thất nghiệp và tăng trưởng GDP ở các nước Ả Rập giai đoạn 1994 - 2010. Kết quả nghiên cứu chỉ ra rằng tăng trưởng kinh tế có quan hệ tiêu cực và đáng kể với tỷ lệ thất nghiệp, điều này là phù hợp với Định luật Okun.

Chu (2008) thực hiện một nghiên cứu thực nghiệm phân tích mối quan hệ giữa tăng trưởng kinh tế và tỷ lệ thất nghiệp ở tỉnh Quảng Đông (Trung Quốc) giai đoạn 1978 - 2016. Kết quả nghiên cứu cho thấy có một mối quan hệ tích cực và ổn định trong dài hạn giữa tỷ lệ thất nghiệp và tăng trưởng kinh tế, cụ thể, tăng trưởng kinh tế tăng $1 \%$ dẫn đến tỷ lệ thất nghiệp tăng lên $0.22 \%$. Bên cạnh đó, tồn tại quan hệ nhân quả hai chiều giữa tăng trưởng kinh tế và tỷ lệ thất nghiệp, nghĩa là, tỷ lệ thất nghiệp dẫn đến sự thay đổi trong tăng trưởng kinh tế và tăng trưởng kinh tế cũng dẫn đến sự thay đổi trong tỷ lệ thất nghiệp.

Mosikari (2013) sử dụng kiểm định đồng liên kết Johansen và kiểm định nhân quả Granger đánh giá ảnh hưởng của tỷ lệ thất nghiệp đến tổng sản phẩm quốc nội ở Nam Phi giai đoạn 1980 - 2011. Kết quả nghiên cứu tìm thấy mối quan hệ dài hạn, nhưng không tìm thấy quan hệ nhân quả Granger giữa tỷ lệ thất nghiệp và tăng trưởng kinh tế ở Nam Phi.

Göçer và Erdal (2015) sử dụng phương pháp phân tích dữ liệu bảng để nghiên cứu mối quan hệ giữa tỷ lệ thất nghiệp của thanh niên và tăng trưởng kinh tế ở 18 quốc gia Châu Âu giai đoạn 1996 - 2012 theo Định luật Okun. Nghiên cứu tìm thấy mối quan hệ tiêu cực trong dài hạn giữa tỷ lệ thất nghiệp của thanh niên và tăng trưởng kinh tế, nghĩa là sự phát triển vượt bậc về kinh tế ở một quốc gia sẽ làm giảm tỷ lệ thất nghiệp của thanh niên ở quốc gia đó.

Misini và Pantina (2017) đã sử dụng hồi quy tuyến tính đơn giản để đánh giá mối quan hệ giữa tổng sản phẩm quốc nội (GDP) danh nghĩa (tăng trưởng kinh tế) và tỷ lệ thất nghiệp ở Kosovo. Nghiên cứu cũng tìm thấy mối quan hệ tiêu cực giữa GDP danh nghĩa và tỷ lệ thất nghiệp ở Kosovo.

Noor, Nor, và Ghani (2007) nghiên cứu về mối quan hệ giữa sản lượng quốc gia (tổng sản phẩm quốc nội) và tỷ lệ thất nghiệp ở Malaysia và xác định xem Định luật Okun có tồn tại ở Malaysia không? Sau khi khẳng định các biến có tính dừng ở sai phân bậc nhất, kết quả hồi quy tìm thấy mối quan hệ tiêu cực trong dài hạn và quan hệ nhân quả Granger hai chiều giữa tỷ lệ thất nghiệp và tổng sản phẩm quốc nội (tăng trưởng kinh tế).

Imran, Mughal, Salman, và Makarevic (2015) sử dụng phương pháp hiệu ứng cố định và mô hình hồi quy gộp (the fixed effect and Pooled OLS) để đánh giá mối quan hệ giữa thất nghiệp và tăng trưởng tài khóa của 12 quốc gia châu Á giai đoạn 1982 - 2011. Kết quả nghiên cứu cho thấy có một mối quan hệ tiêu cực đáng kể giữa thất nghiệp và tăng trưởng tài khóa.

Tương tự, Makaringe và Khobai (2018) đánh giá xu hướng và ảnh hưởng của tỷ lệ thất nghiệp lên tăng trưởng tài khóa ở Nam Phi bằng cách sử dụng dữ liệu định kỳ trong giai đoạn từ quý 01 năm 1994 đến quý 04 năm 2016. Nghiên cứu sử dụng mô hình ARDL và tìm thấy mối quan hệ tiêu cực giữa thất nghiệp và tăng trưởng tài khóa trong cả ngắn hạn và dài hạn. Kết quả này ở Nam Phi là phù hợp với lý thuyết của Định luật Okun.

Dritsakis và Stamatiou (2016) sử dụng dữ liệu hàng năm giai đoạn 1995 - 2015 đánh giá mối quan hệ giữa tỷ lệ thất nghiệp, tăng trưởng tài khóa và tỷ lệ lạm phát ở Hi Lạp. Nghiên cứu sử dụng kiểm định $\mathrm{ARDL}$ và mô hình $\mathrm{VECM}$ kiểm tra sự đồng liên kết dài hạn và ngắn hạn và hướng quan hệ nhân quả giữa các biến. Kết quả nghiên cứu cho thấy tồn tại mối quan hệ tiêu cực 
giữa thất nghiệp và tăng trưởng tài khóa trong cả ngắn hạn và dài hạn và quan hệ nhân quả một chiều chạy từ thất nghiệp đến tăng trưởng tài khóa.

Lam (2014) nghiên cứu mối quan hệ giữa tỷ lệ thất nghiệp và tăng trưởng GDP và xem xét Định luật Okun có phù hợp ở Philippines không. Nghiên cứu sử dụng các kiểm định EngleGranger và đồng liên kết Johansen để xem xét mối quan hệ dài hạn giữa các biến và kết quả của cả hai kiểm định này đều cho thấy tồn tại mối quan hệ dài hạn giữa thất nghiệp và tăng trưởng GDP ở Philippines. Sau đó, kiểm tra quan hệ nhân quả Granger được tiến hành và chỉ tìm thấy hướng của mối quan hệ nhân quả chạy từ thất nghiệp đến tăng trưởng GDP, đồng nghĩa với việc Định luật Okun là phù hợp ở Philippines.

Nhìn chung, các nghiên cứu chỉ ra những phát hiện mâu thuẫn nhau về mối quan hệ giữa tỷ lệ thất nghiệp với tăng trưởng kinh tế. Định luật Okun khẳng định tồn tại mối quan hệ nghịch biến giữa thất nghiệp và tăng trưởng kinh tế, trong khi các nghiên cứu thực nghiệm đồng thuận với quan điểm Định luật Okun và tìm thấy mối quan hệ tiêu cực giữa thất nghiệp và tăng trưởng kinh tế ở một số quốc gia trong ngắn hạn hoặc dài hạn hoặc cả ngắn hạn và dài hạn. Tuy nhiên, vẫn có một số phát hiện thực nghiệm khác phủ nhận Định luật Okun vì kết quả nghiên cứu cho thấy mối quan hệ tích cực hoặc không có mối quan hệ nào giữa thất nghiệp và tăng trưởng kinh tế.

\section{Phương pháp nghiên cứu}

\subsection{Nguồn dũ liệu}

Nghiên cứu sử dụng dữ liệu chuỗi thời gian hàng năm của biến tăng trưởng kinh tế đại diện bởi tổng sản phẩm quốc nội (GDP thực tế Việt Nam theo giá cố định năm 2010) được thu thập từ dữ liệu của World Bank (n.d.) và thất nghiệp (\% so với tổng lực lượng lao động) được thu thập từ Niêm giám thống kê của Tổng cục thống kê Việt Nam (n.d.) giai đoạn 1986 - 2019 (giai đoạn từ sau Đổi mới).

\subsection{Mô hình nghiên cứu cụ thể}

Nghiên cứu dựa trên mô hình của Okun (1962) được trình bày bởi Ademola và Badiru (2016), trong đó Tổng sản phẩm quốc nội (GDP) thực tế đại diện cho tăng trưởng kinh tế là biến độc lập và tỷ lệ thất nghiệp (tỷ lệ số người hiện không có việc làm, nhưng đang tìm việc so với tổng lực lượng lao động) là biến phụ thuộc. Mô hình cụ thể như sau:

$$
\mathrm{Y}=\alpha_{0}+\alpha_{1} \mathrm{U}_{\mathrm{t}}+\varepsilon_{\mathrm{t}}
$$

Trong đó, Y là tỷ lệ thất nghiệp, U là tăng trưởng kinh tế (GDP thực tế). Sửa đổi ký hiệu mô hình (2) như sau:

$$
\text { Unempl }=\alpha_{0}+\alpha_{1} \operatorname{Rgdp}+\varepsilon_{\mathrm{t}}
$$

Trong đó, $R g d p$ là tổng sản phẩm quốc nội (GDP) thực tế; Unempl là tỷ lệ thất nghiệp; $\alpha_{1}$ là tham số; $\varepsilon_{\mathrm{t}}$ - error term là thuật ngữ lỗi (nhiễu trắng)

Do GDP thực tế $(R g d p)$ được đo lường bởi đơn vị tiền tệ, trong khi tỷ lệ thất nghiệp (Unempl) được đo lường bởi tỷ lệ phần trăm, phương trình (3) được biến đổi thành dạng ước lượng như sau:

$$
\text { Lnunempl }=\alpha_{1}+\alpha_{2} \operatorname{Lnrgdp}+\varepsilon_{\mathrm{t}}
$$

\subsection{Phương pháp thục nghiệm}

\subsubsection{Kiểm định gốc đơn vị (Unit Root Test)}

Kiểm định gốc đơn vị là bước quan trọng đầu tiên trong ước lượng mô hình. Bước này được thực hiện trên các biến (thất nghiệp và tăng trưởng kinh tế) để kiểm tra xem các biến là có 
tính dừng ở gốc đơn vị (ký hiệu $\mathrm{I}(0))$ hay có tính dừng ở sai phân bậc 01 (I(1)) vì nếu không kiểm tra tính dừng, đôi khi nghiên cứu sẽ cho kết quả hồi quy giả nếu dữ liệu chuỗi dừng được hồi quy trên một dữ liệu không dừng khác (Gujarati, 2004).

Nghiên cứu sử dụng cả kiểm định gốc đơn vị Augmented Dickey-Fuller (ADF) của Dickey và Fuller (1979) và kiểm định gốc đơn vị PhillipsPerron $(\mathrm{PP})$ của Phillips và Perron (1988) để kiểm tra tính dừng của các biến. Việc chấp nhận hay bác bỏ giả thuyết không $\left(\mathrm{H}_{0}\right)$ : dữ liệu nghiên cứu là không dừng phụ thuộc vào kiểm định $\mathrm{t}$ của độ trễ và thống kê $\mathrm{t}$. Nếu kiểm định $\mathrm{t}$ của độ trễ là một lượng nhỏ hơn điểm tới hạn thì giả thuyết không $\left(\mathrm{H}_{0}\right)$ về sự hiện diện của gốc đơn vị được chấp nhận.

\subsubsection{Kiểm định đồng liên kết độ trễ phân phối tụ hồi quy (ARDL)}

Kiểm định đồng liên kết $\mathrm{ARDL}$ là bước tiếp theo của quy trình ước lượng. Bước này được thực hiện để giải thích mối quan hệ dài hạn giữa các biến của nghiên cứu. Hầu hết các tài liệu trong lĩnh vực kinh tế học đã sử dụng phương pháp đồng liên kết Johansen trong việc ước lượng mối quan hệ dài hạn giữa các biến vì đây được xem là cách tốt nhất khi xử lý các biến I(1). Bên cạnh đó, các nghiên cứu gần đây đã giới thiệu Kiểm định đồng liên kết ARDL như là một giải pháp thay thế trong nỗ lực giải quyết vấn đề đồng liên kết và có rất nhiều lợi ích vượt trội hơn so với phương pháp đồng liên kết Johansen. Thứ nhất, kiểm định ARDL tương đối hiệu quả hơn khi kích thước dữ liệu của nghiên cứu nhỏ. Dữ liệu nghiên cứu bao gồm 33 quan sát là khá tốt nếu xét về phạm vi và tính chất của nghiên cứu. Thư hai, kiểm định ARDL cho phép kỹ thuật bình phương nhỏ nhất (OLS) thông thường ước lượng sự đồng liên kết khi độ trễ của mô hình được xác định. Điều này làm cho phương pháp ARDL trở thành mô hình tốt nhất trong trường hợp này. Cuối cùng, kiểm định ARDL có thể giải quyết mô hình khi các biến có tính dừng hỗn hợp. Cụ thể, chúng ta có thể áp dụng kỹ thuật Johansen khi các biến của nghiên cứu là dừng ở mức $\mathrm{I}(0)$ nhưng khi tất cả các biến đều dừng ở $(\mathrm{I}(1))$ hoặc các biến dừng ở cả $\mathrm{I}(0)$ và $\mathrm{I}(1)$, mô hình $\mathrm{ARDL}$ là lựa chọn tối ưu. Do đó, mô hình $\mathrm{ARDL}$ có thể không cần kiểm tra tính dừng của các biến trước mà chỉ cần sử dụng hai bước trong ước lượng của nó đó là: Kiểm định $\mathrm{F}$ để xác định mối quan hệ dài hạn và ước lượng mô hình sửa lỗi (ECM) để kiểm tra mối quan hệ ngắn hạn.

\subsubsection{Kiểm định đường bao ARDL (ARDL Bounds test)}

Kiểm định này được thực hiện theo hai quy trình chính. Quy trình đầu tiên là ước lượng phương trình $\mathrm{ARDL}$ bằng cách sử dụng công cụ ước lượng bình phương nhỏ nhất thông thường khác để kiểm tra xem liệu có tồn tại mối quan hệ dài hạn giữa các biến của nghiên cứu hay không. Sau đó, kiểm định $\mathrm{F}$ được tiến hành cho mức ý nghĩa kết hợp đối với các hệ số co giãn của các biến ở trạng thái trễ của chúng. Giả thuyết cho kiểm định là:

\section{$H_{0}=\partial_{0}$ (không tồn tại mối quan hệ giũa các biến)}

\section{$H_{1} \neq \partial_{0}$ (tồn tại mối quan hệ giũua các biến)}

Các giá trị tới hạn dẫn đến kiểm định đồng liên kết khi các biến của nghiên cứu là dừng ở gốc đơn vị $(\mathrm{I}(0))$ hoặc dừng ở sai phân bậc 01 (I(1)). Có một giả định về giá trị giới hạn dưới trong đó thứ tự của sự kết hợp của biến giải thích là 0 , hoặc $\mathrm{I}(0)$ với thứ tự của sự tích hợp của giới hạn trên là $01, \mathrm{I}(1)$. Khi giá trị tính toán $\mathrm{F}$ lớn hơn giới hạn trên, có thể kết luận rằng có sự đồng liên kết giữa hai biến của nghiên cứu, nghĩa là giả thuyết không $\left(\mathrm{H}_{0}\right)$ của không có mối quan hệ giữa các biến bị bác bỏ. Ngược lại, khi giá trị tính toán $\mathrm{F}$ nằm giữa giới hạn dưới và giới hạn trên, không thể kêt luận được các biến có đồng liên kết hay không. Về mặt thứ bậc, cần phải tiến hành kiểm định đường bao, do đó chúng ta áp đặt một giới hạn đối với ARDL để ước lượng mối quan hệ dài hạn giữa các biến phụ thuộc và độc lập của nghiên cứu. 


\subsubsection{Kiểm tra quan hệ nhân quả}

Kiểm tra quan hệ nhân quả là bước cuối cùng của quy trình ước lượng. Nó được thực hiện để kiểm tra mối quan hệ nhân quả giữa tỷ lệ thất nghiệp và tăng trưởng kinh tế. Các nghiên cứu thường sử dụng kiểm định Engle và Granger (1989) và kiểm định nhân quả Granger để biết mối quan hệ nhân quả giữa hai biến của nghiên cứu, liệu một biến này trực tiếp gây ra biến còn lại hay các biến không ảnh hưởng đến nhau.

\section{Kết quả nghiên cứu}

\subsection{Kiểm định gốc đơn vị (Unit root test)}

Nghiên cứu sử dụng các kiểm định gốc đơn vị $\mathrm{ADF}$ và $\mathrm{PP}$ để xem xét tính dừng trong các biến của nghiên cứu. Ý nghĩa của thống kê này là nếu dữ liệu của biến tương ứng có gốc đơn vị, thì biến đó không dừng và ngược lại.

\section{Bảng 1}

Kết quả của kiểm định gốc đơn vị Augmented Dickey-Fuller và Phillips-Perron

\begin{tabular}{lcccccccc}
\hline \multicolumn{1}{c}{ Biến } & $\begin{array}{c}\text { ADF } \\
\text { chuỗi gốc }\end{array}$ & $\begin{array}{c}\text { PP chuỗi } \\
\text { gốc }\end{array}$ & $\begin{array}{c}\text { Giá trị tới } \\
\text { hạn }\end{array}$ & $\begin{array}{c}\text { ADF chuỗi sai } \\
\text { phân bậc 1 }\end{array}$ & $\begin{array}{c}\text { PP chuỗi sai } \\
\text { phân bậc 1 }\end{array}$ & $\begin{array}{c}\text { Giá trị tới } \\
\text { hạn }\end{array}$ & $\begin{array}{c}\text { Cấp độ của } \\
\text { tích họ̣p }\end{array}$ \\
\hline Lnrgdp & -2.227058 & -2.899145 & -3.557759 & -3.855217 & -3.868908 & $-3.557759^{*}$ & $\mathrm{I}(1)^{*}$ \\
\hline Lnunempl & 0.112151 & -0.004954 & -1.951332 & -9.881987 & -9.837821 & $-1.951687^{*}$ & $\mathrm{I}(1)^{*}$ \\
\hline
\end{tabular}

Ghi chú: * biểu thị sự bác bỏ giả thuyết không về gốc đơn vị ở mức ý nghĩa $5 \%$. Các giá trị tới hạn tương ứng cho các kiểm định gốc đơn vị ADF và PP được thu thập từ các giá trị một phía của MacKinnon (1996)

Nguồn: Tính toán của tác giả bằng phần mềm Eviews 10

Đối với cả kiểm định gốc đơn vị $\mathrm{ADF}$ và $\mathrm{PP}$, giá trị tuyệt đối của giá trị thống kê kiểm tra của các biến nhỏ hơn các giá trị tới hạn tương ứng của nó ở chuỗi gốc, do đó chúng ta không thể bác bỏ giả thuyết không $\left(\mathrm{H}_{0}\right)$ về sự hiện diện của gốc đơn vị đối với các biến ở chuỗi gốc. Tuy nhiên, khi kiểm tra tính dừng chuỗi dữ liệu tỷ lệ thất nghiệp và tăng trưởng kinh tế (GDP) ở sai phân bậc 1 ở mức ý nghĩa $5 \%$, các chuỗi dữ liệu là có tính dừng. Do đó, chuỗi dữ liệu của các biến là phù hợp để sử dụng trong nghiên cứu này.

\subsection{Kiểm định đưòng bao ARDL (ARDL Bounds test)}

Kiểm định đường bao nhằm mục đích kiểm tra mối quan hệ dài hạn giữa tăng trưởng kinh tế (GDP) và tỷ lệ thất nghiệp.

\section{Bảng 2}

Kết quả của kiểm định đường bao ARDL

\section{Kiểm tra sự tồn tại của mối quan hệ dài hạn giữa các biến trong mô hình ARDL}

\begin{tabular}{|c|c|c|c|c|}
\hline $\mathbf{K}$ & $95 \%$ giới hạn dưới & 95\% giới hạn trên & $90 \%$ giới hạn dưới & $90 \%$ giới hạn trên \\
\hline 1 & 4.94 & 5.73 & 4.04 & 4.78 \\
\hline Mô hình & & Giá trị F tính toán & & Quan hệ đồng liên \\
\hline $\begin{array}{c}\text { Lnrgdp } \\
\text { (Lnunempl) }\end{array}$ & & 20.12735 & & kết suy luậ \\
\hline
\end{tabular}

Nguồn: Tính toán của tác giả bằng phần mềm Eviews 10 
Từ kết quả Bảng 2 ta thấy, giá trị thống kê $\mathrm{F}$ được tính toán (20.12735) lớn hơn cả mức tin cậy giới hạn trên $95 \%$ và $90 \%$ là 5.73 và 4.78 tương ứng. Do đó, có thể khẳng định rằng có quan hệ đồng liên kết giữa biến phụ thuộc (tỷ lệ thất nghiệp) và biến độc lập (tăng trưởng kinh tế (GDP)). Kết quả nghiên cứu là giống với các nghiên cứu của Soylu và cộng sự (2017) ở các nước Đông Âu, Banda và cộng sự (2016) và Mosikari (2013) ở các nước Nam Phi.

\subsection{Kết quả mô hình ARDL về mối quan hệ dài hạn}

Mối quan hệ dài hạn giữa biến phụ thuộc (tỷ lệ thất nghiệp) và biến độc lập (tăng trưởng kinh tế (GDP)) được ước lượng bằng mô hình ADRL. Độ co giãn trong dài hạn được biểu thị bằng các hệ số của biến phụ thuộc (tỷ lệ thất nghiệp).

\section{Bảng 3}

Ước lượng mô hình thất nghiệp dài hạn

\begin{tabular}{ccc}
\hline & \multicolumn{3}{c}{ Biến phụ thuộc: Lnunempl } \\
\hline Hồi quy & \multicolumn{2}{c}{ Autoregressive Distributed Lag Model } \\
\hline Hnệ số co giãn dài hạn & Giá trị thống kê t \\
\hline C & -0.094702 & $(-2.301097)^{*}$ \\
\hline
\end{tabular}

Ghi chú: * biểu thị mức ý nghĩa 5\%. Giá trị trong ngoặc là thống kê t. ARDL (1.4) dựa trên tiêu chí Swchwarz Bayesian Nguồn: Tính toán của tác giả bằng phần mềm Eviews 10

Dựa trên kết quả Bảng 3 ở trên, hệ số co giãn dài hạn của biến phụ thuộc (tỷ lệ thất nghiệp) là tiêu cực và có ý nghĩa thống kê ở mức ý nghĩa $5 \%$. Cụ thể, tăng trưởng kinh tế tăng $1 \%$ sẽ làm giảm tỷ lệ thất nghiệp $0.09 \%$. Kết quả này là phù hợp với Định luật Okun và giống với các kết quả nghiên cứu của Chu (2008) và Li và Liu (2012) ở Trung Quốc, và Haririan và cộng sự (2010) ở các quốc gia MENA, đồng thời khẳng định tồn tại mối quan hệ dài hạn giữa tăng trưởng kinh tế (GDP) và thất nghiệp ở Việt Nam.

\subsection{Kết quả của mô hinh sửa lỗi (ECM) trong ngắn hạn}

Việc ước lượng Mô hình sửa lỗi $(\mathrm{ECM})$ ngắn hạn trở nên bắt buộc khi tồn tại mối quan hệ dài hạn giữa các biến. Mô hình ECM đo lường sự năng động của mô hình ngắn hạn và tốc độ mà mô hình này được điều chỉnh về trạng thái cân bằng bất cứ khi nào có một cú sốc xảy ra. Mô hình ECM thực hiện ở sai phân bậc nhất được trình bày kết quả ở Bảng 4.

\section{Bảng 4}

Ước lượng mô hình sửa lỗi (ECM) ngắn hạn sử dụng phương pháp ARDL

\section{Biến phụ thuộc: D(Lnunempl)}

\begin{tabular}{ccc} 
Hồi quy & \multicolumn{2}{c}{ Autoregressive Distributed Lag Model } \\
& Hệ số co giãn ngắn hạn & Giá trị thống kê t \\
Lnrgdp & -0.573566 & $(-3.793270)^{*}$ \\
\hline $\mathrm{C}$ & 0.853731 & $(3.640114)$ \\
\hline
\end{tabular}

Ghi chú: * biểu thị mức ý nghĩa 5\%. Giá trị trong ngoặc là thống kê t. ARDL (1.4) dựa trên tiêu chí Swchwarz Bayesian Nguồn: Tính toán của tác giả bằng phần mềm Eviews 10 
Từ Bảng 4 ở trên, hệ số co giãn ngắn hạn của tăng trưởng kinh tế (GDP) là âm và có ý nghĩa thống kê ở mức ý nghĩa $5 \%$. Điều này có nghĩa là tăng trưởng kinh tế (GDP) có mối quan hệ tiêu cực trong ngắn hạn với tỷ lệ thất nghiệp và tăng trưởng kinh tế (GDP) tăng $1 \%$ sẽ làm tỷ lệ thất nghiệp giảm $0.57 \%$. Kết quả nghiên cứu này là giống với nghiên cứu của Makaringe và Khobai (2018) ở các nước Nam Phi, Dritsakis và Stamatiou (2016) ở Hi Lạp và Lam (2014) ở Philippines.

\subsection{Kết quả quan hệ nhân quả Granger}

Kiểm định gốc đơn vị $\mathrm{ADF}$ và $\mathrm{PP}$ chỉ ra rằng tăng trưởng kinh tế (GDP) và tỷ lệ thất nghiệp là có tính dừng ở sai phân bậc nhất $\mathrm{I}(1)$. Do đó, nghiên cứu đã sử dụng sự khác biệt $\log$ sai phân bậc nhất giữa các biến khi tiến hành kiểm tra nhân quả Granger.

\section{Bảng 5}

Kết quả của kiểm tra nhân quả Granger

\begin{tabular}{ccc}
\hline \multicolumn{1}{c}{ Giả thuyết không } & Giá trị thống kê F & Xác suất \\
\hline Lnunempl không dẫn đến sự thay đổi của Lnrgdp & 1.13812 & 0.3353 \\
Lnrgdp không dẫn đến sự thay đổi của Lnunempl & 1.83056 & 0.1797 \\
\hline
\end{tabular}

Nguồn: Tính toán của tác giả bằng phần mềm Eviews 10

Kết quả Bảng 5 cho thấy, thất nghiệp không dẫn đến sự thay đổi của tăng trưởng kinh tế (GDP) và tăng trưởng kinh tế (GDP) cũng không dẫn đến sự thay đổi của tỷ lệ thất nghiệp ở Việt Nam vì các giá trị thống kê $F$ là không có ý nghĩa. Các kết quả nghiên cứu này ủng hộ nghiên cứu của Mosikari (2013) ở các nước Nam Phi là không có mối quan hệ nhân quả giữa tăng trưởng kinh tế (GDP) và thất nghiệp.

\section{Kết luận}

Nghiên cứu này tập trung vào đánh giá mối quan hệ giữa thất nghiệp và tăng trưởng kinh tế ở Việt Nam. Dữ liệu nghiên cứu là dữ liệu chuỗi thời gian giai đoạn 1986 - 2019 được thu thập thông qua Niêm giám thống kê của Tổng cục thống kê Việt Nam (n.d.). Các kiểm định đường bao, kiểm định $\mathrm{ARDL}$ và kiểm định $\mathrm{ECM}$ chỉ ra rằng tỷ lệ thất nghiệp có mối quan hệ tiêu cực với tăng trưởng kinh tế Việt Nam trong ngắn hạn và dài hạn. Tuy nhiên, không tồn tại mối quan hệ nhân quả giữa hai biến, điều này có thể là do các yếu tố bên ngoài khác có thể đã gây ra mối quan hệ giữa tỷ lệ thất nghiệp và tăng trưởng kinh tế trong cả dài hạn và ngắn hạn và bản thân các biến bên ngoài đó không có quan hệ.

Tỷ lệ thất nghiệp và tốc độ tăng trưởng kinh tế có mối quan hệ tiêu cực trong ngắn hạn và dài hạn. Vì vậy, để giảm tỷ lệ thất nghiệp ở Việt Nam, cần thúc đẩy tăng trưởng kinh tế bằng cách khơi dậy sự mong muốn tham gia vào các hoạt động kinh tế của những người lao động trẻ (thanh niên) hoặc buộc thôi việc những người đã đến tuổi nghỉ hưu mà chưa muốn nghỉ hưu để nhường chỗ cho những thanh niên thất nghiệp tham gia vào thị trường lao động, điều này sẽ góp phần tăng trưởng kinh tế.

Bên cạnh đó, để thúc đẩy tăng trưởng kinh tế và giảm tỷ lệ thất nghiệp, chính phủ Việt Nam cần có những chính sách hợp lý như nới lỏng các chính sách tài chính, cải cách thủ tục hành chính và pháp lý nhằm khuyến khích đầu tư nước ngoài vào trong nước, khuyến khích phát triển các doanh nghiệp vừa và nhỏ, giảm thuế và cho các doanh nghiệp này vay vốn để mở rộng quy mô sản xuất, ưu đãi đối với doanh nghiệp thuộc mọi thành phần kinh tế tham gia đầu tư các 
dự án, công trình có quy mô lớn ... điều này gián tiếp giúp đỡ và kích thích các doanh nghiệp sử dụng nhiều lao động hơn. Thêm vào đó, ở Việt Nam, lao động ở khu vực nông nghiệp chiếm tỷ trọng lớn, do đó cần hỗ trợ ngành nông nghiệp bằng cách thay đổi cách tiếp cận đầu tư công, đa dạng hóa sản xuất nông nghiệp, tạo cơ hội cho nông dân, người nghèo được trực tiếp tham gia vào các hoạt động kinh tế ... Ngoài ra, chính phủ cần chuyển dịch cơ cấu lao động theo hướng công nghiệp và dịch vụ, đào tạo nghề nâng cao trình độ cho lao động nông thôn, hướng người lao động đến những việc làm ở những ngành nghề đem lại giá trị cao trong các chuỗi giá trị, tạo ra một thị trường lao động cạnh tranh để giảm nguy cơ thất nghiệp khi có khủng hoảng.

Nghiên cứu này sẽ tiếp tục phát triển các nghiên cứu tiếp theo sâu rộng hơn bằng cách thêm vào mô hình thực nghiệm các yếu tố kinh tế vĩ mô khác như lạm phát, lãi suất, mức cung tiền và tỷ giá hối đoái có thể tồn tại mối quan hệ với tăng trưởng kinh tế Việt Nam trong cả ngắn hạn và dài hạn.

\section{Tài liệu tham khảo}

Adarkwa, S., Donkor, F., \& Kyei, E. (2017). The impact of economic growth on unemployment in Ghana: Which economic sector matters most? The International Journal of Business \& Management, 5(4), 1-3.

Ademola, A. S., \& Badiru, A. (2016). The impact of unemployment and inflation on economic growth in Nigeria (1981 - 2014). International Journal of Business and Economic Sciences Applied Research, 9(1), 47-55.

Anghel, M. G., Anghelache, C., \& Manole, A. (2017). The effect of unemployment on economic growth. Romanian Statistical Review, 65(7), 174-186.

Banda, H., Ngirande, H., \& Hogwe, F. (2016). The impact of economic growth on unemployment in South Africa: 1994 - 2012. Investment Management and Financial Innovations, 13(2), 246-255.

Chu, Z. H. (2008). An empirical analysis of the relationship between economic growth and employment in Guangdong. Journal of South China Normal University (Social Science Edition), 2008(2), 15-22.

Daly, M., \& Hobijn, B. (2010). Okun's law and the unemployment surprise of 2009. FRBSF Economic Letter, 8, 1-4.

Dfid, G. B. (2008). Growth: Building jobs and prosperity in developing countries. London, UK: Department for International Development.

Dickey, D. A., \& Fuller, W. A. (1979). Distribution of the estimators for autoregressive time series with a unit root. Journal of the American Statistical Association, 74(366), 427-431.

Dritsakis, N., \& Stamatiou, P. (2016). The effects of unemployment on economic growth in Greece: An ARDL Bound test approach. Romanian Journal of Economic Forecasting, 19(62), 53-72.

Enejoh, S. Y., \& Tsauni, A. M. (2017). An analytical study of the impact of unemployment on economic growth in Nigeria (1970 - 2016). IOSR Journal of Humanities and Social Science, 22(11), 74-81.

Engle, R. F., \& Granger, C. W. J. (1989). Cointegration and error correction: Representation, estimation and testing. Econometrica, 55(2), 251-276. 
Göçer, İ., \& Erdal, L. (2015). The relationship between youth unemployment and economic growth in central and eastern European countries: An empirical analysis. Journal of the Faculty of Economics and Administrative Sciences (Çankırl Karatekin University), 5(1), 173-188.

Gujarati, D. N. (2004). Basic econometrics (4th ed.). New York, NY: McGraw-Hill Higher Education.

Gyang, E. J., Anzaku, P. E., \& Iyakwari, A. D. (2018). An analysis of the relationship between unemployment, inflation and economic growth in Nigeria: 1986 - 2015. Bingham Journal of Economics and Allied Studies, 1(1), 225-233.

Habees, M. A. A., \& Rumman, M. A. (2012). The relationship between unemployment and economic growth in Jordan and some Arab countries. World Applied Sciences Journal, $18(5), 673-680$.

Haririan, M., Bilgin, M. H., \& Karabulut, G. (2010). The relationship between GDP and unemployment: Evidence from MENA Countries. Zagreb International Review of Economics and Business, 13(1), 17-28.

Imran, M., Mughal, K. S., Salman, A., \& Makarevic, N. (2015). Unemployment and economic growth of developing Asian countries: A panel data analysis. European Journal of Economic Studies, 13(3), 147-160.

Jhingan, M. L. (2003). Advanced macroeconomics theory (11th ed.). Delhi, India: Vrinda Publication Ltd.

Khaliq, S., Soufan, T., \& Shihab, R. A. (2014). The relationship between unemployment and economic growth rate in Arab country. Journal of Economics and Sustainable Development, 4(7), 56-59.

Kreishan, F. M. (2011). Economic growth and unemployment: An empirical analysis. Journal of Social Sciences, 7(2), 228-231.

Lam, J. J. (2014). The applicability of Okun's relationship between unemployment and GDP growth in the Philippines: A time series approach. Manila, Philippines: School of Economics, De La Salle University.

Levine, L. (2013). Economic growth and the unemployment rate. Retrieved May 10, 2020, from the website of Federation of American Scientists https://fas.org/sgp/crs/misc/R42063.pdf

Li, C., \& Liu, Z. (2012). Study on the relationship among Chinese unemployment rate, economic growth and inflation. Advance in Applied Economics and Finance, 1(1), 1-6.

MacKinnon, J. G. (1996). Numerical distribution functions for unit root and cointegration tests. Journal of Applied Econometrics, 11, 601-618.

Makaringe, S. C., \& Khobai, H. (2018). The effect of unemployment on economic growth in South Africa (1994 - 2016). Retrieved May 15, 2020, from https://mpra.ub.unimuenchen.de/85305/1/MPRA_paper_85305.pdf

Misini, S., \& Pantina, M. B. (2017). The effect of economic growth in relation to unemployment. Journal of Economics and Economic Education Research, 18(2), 1-9.

Mosikari, T. J. (2013). The effect of unemployment rate on Gross Domestic Product: Case of South Africa. Mediterranean Journal of Social Sciences, 4(6), 429-434. 
Nguyen, Q. H. (2016). Relationship between economic growth, unemployment and poverty: Analysis at provincial level in Vietnam. International Journal of Economics and Finance, 8(12), 113-119.

Noor, Z. M., Nor, N. M., \& Ghani, J. A. (2007). The relationship between output and unemployment in Malaysia: Does Okun's law exist? International Journal of Economics and Management, 1(3), 337-344.

Okun, A. M. (1962). Potential GNP: Its measurement and significance. Proceedings of the business and economics statistics section (pp. 98-104). New Haven, USA: Yale University, Cowles Foundation for Research in Economics.

Özel, H. A., Sezgin, F. H., \& Topkaya, Ö. (2013). Investigation of economic growth and unemployment relationship for G7 countries using Panel regression analysis. International Journal of Business and Social Science, 4(6), 163-171.

Phillips, P. C. B., \& Perron, P. (1988). Testing for a unit root in time series regression. Biometrika, 75(2), 335-346.

Piketty, T. (2014). Capital in the twenty-first century. Cambridge, UK: The Belknap Press of Harvard University Press.

Schiliro, D. (2017). A glance at Solow's growth theory. Journal of Mathematical Economics and Finance, 3(2(5), 83-103.

Seth, A., John, M. A., \& Dalhatu, A. Y. (2018). The impact of unemployment on economic growth in Nigeria: An application of Autoregressive Distributed Lag (ARDL) bound testing. Sumerianz Journal of Business Management and Marketing, 1(2), 37-46.

Solow, R. M. (1956). A contribution to the theory of economic growth. The Quarterly Journal of Economics, 70(1), 65-94.

Soylu, Ö. B., Çakmak, İ., \& Okur, F. (2017). Economic growth and unemployment issue: Panel data analysis in Eastern European countries. Journal of International Studies, 11(1), 93-107.

Tổng cục thống kê Việt Nam. (n.d.). Niêm giám thống kê qua các năm [Statistical yearbook over the years]. Hanoi, Vietnam: Nhà xuất bản Thống kê.

World Bank. (n.d.). GDP (constant 2010 US\$) - Vietnam indicators. Retrieved November 18, 2020, from https://data.worldbank.org/indicator/NY.GDP.MKTP.KD?locations=VN

Yilmaz, Ö. G. (2005). Türkiye ekonomisinde büyüme ile işsizlik oranları arasındaki nedensellik ilişkisi [The causality relationship between growth and unemployment rates in the Turkish economy]. Ekonometri ve Istatistik e-Dergisi, 2, 63-76.

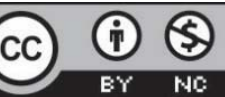

Creative Commons Attribution-NonCommercial 4.0 International License. 